\title{
Article \\ Quantification of Venetoclax for Therapeutic Drug Monitoring in Chinese Acute Myeloid Leukemia Patients by a Validated UPLC-MS/MS Method
}

\author{
Xi Yang $\left.{ }^{1}{ }^{(}\right)$, Chen $\mathrm{Mei}^{2}$, Xiaoying He ${ }^{1}$, Lingjuan $\mathrm{He}^{1}{ }^{1}$, Xiaoyang $\mathrm{Lu}^{1}$, Hongyan Tong ${ }^{2, *}$ and Yan Lou ${ }^{1, *}$ \\ 1 Key Laboratory for Drug Evaluation and Clinical Research of Zhejiang Province, Department of Clinical \\ Phamacy, The First Affiliated Hospital, College of Medicine, Zhejiang University, 79 QingChun Road, \\ Hangzhou 310000, China; 1516100@zju.edu.cn (X.Y.); hxy17858505013@163.com (X.H.); \\ hlingj19@163.com (L.H.); luxiaoyang@zju.edu.cn (X.L.) \\ 2 Department of Hematology, The First Affiliated Hospital, College of Medicine, Zhejiang University, \\ 79 QingChun Road, Hangzhou 310000, China; meichenblood@yeah.net \\ * Correspondence: tonghongyan@zju.edu.cn (H.T.); yanlou@zju.edu.cn (Y.L.); \\ Tel./Fax: +86-571-8723-6675 (H.T. \& Y.L.)
}

\section{check for}

updates

Citation: Yang, X.; Mei, C.; He, X.;

He, L.; Lu, X.; Tong, H.; Lou, Y.

Quantification of Venetoclax for Therapeutic Drug Monitoring in Chinese Acute Myeloid Leukemia Patients by a Validated UPLC-MS/ MS Method. Molecules 2022, 27, 1607. https://doi.org/10.3390/

molecules27051607

Academic Editor: Fernandez De

Simon Brigida

Received: 23 December 2021

Accepted: 22 February 2022

Published: 28 February 2022

Publisher's Note: MDPI stays neutral with regard to jurisdictional claims in published maps and institutional affiliations.

Copyright: (c) 2022 by the authors. Licensee MDPI, Basel, Switzerland. This article is an open access article distributed under the terms and conditions of the Creative Commons Attribution (CC BY) license (https:/ / creativecommons.org/licenses/by/ $4.0 /)$.

\begin{abstract}
Venetoclax has emerged as a breakthrough for treatment of leukemia with a wide interindividual variability in pharmacokinetics. Herein, a rapid, sensitive, and reliable UPLC-MS/MS method for quantification of venetoclax in plasma was developed and validated. The method was operated in the multiple-reaction monitoring (MRM) mode to detect venetoclax at $\mathrm{m} / \mathrm{z}$ transition $868.5>321.0$ and IS at $875.5>321.0$, respectively. Protein precipitation prior to injection into the LC-MS/MS and the analyte was separated on an ACQUITY UPLC BEH C18 column by gradient elution with acetonitrile and $0.1 \%$ formic acid in water. Linear calibration curves were obtained in the range of 25-8000 ng/mL. The specificity, recovery, matrix effect, and stability also met the acceptance criteria of FDA guidance. The method was successfully applied to analyze plasma in acute myeloid leukemia (AML) patients. The peak plasma concentration $\left(C_{\max }\right)$ of venetoclax in Chinese AML patient was $2966.0 \pm 1595.0 \mathrm{ng} / \mathrm{mL}$ while the trough concentration $\left(C_{\min }\right)$ was $1018.0 \pm 729.4 \mathrm{ng} / \mathrm{mL}$. Additionally, $\mathrm{C}_{\max }$ and $\mathrm{C}_{\min }$ showed a positive correlation with AST levels. Furthermore, $\mathrm{C}_{\max }$ was significantly higher in the older patients. The present method can be applied for TDM of venetoclax in treatment of hematological cancers.
\end{abstract}

Keywords: venetoclax; UPLC-MS/MS; therapeutic drug monitoring

\section{Introduction}

Hematological malignancy constitutes serious, high-relapse, and hardly curable malignant tumors. It is characterized by an unchecked proliferation and blocked differentiation of abnormal hematopoiesis stem and progenitor cells, which is accompanied by an obstructed apoptosis [1]. The conventional chemotherapy is unsuitable for treatment in the elderly or patients who cannot tolerate it and chemotherapy resistance is a major cause of treatment failure $[2,3]$. In recent years, targeted therapies in certain aspects of leukemia cell processes such as proliferation and apoptosis brought the improvement of new strategies to treat hematological malignancy [2]. The B cell leukemia/lymphoma-2 (BCL-2), an intrinsic anti-apoptotic protein, is aberrantly overexpressed in leukemia stem cells. It plays a vital role in obstruction of leukemia cell apoptosis and drug resistance [4-6].

Venetoclax is an excellent orally selective inhibitor via competing for the $\mathrm{BH} 3$ binding site on BCL-2, thereby changing the mitochondrial outer membrane permeability, activating the caspase, and then restoring the malignant cells' apoptosis pathway [7,8]. In April 2016, the FDA granted accelerated approval to venetoclax as monotherapy for treatment in patients diagnosed with CLL /SLL with chromosome 17p deletion who have received at least one prior therapy [9]. Subsequently, the FDA expanded venetoclax approval in 
combination with hypomethylating agent drugs (HMAs) for AML patients who are at least 75 years old or unsuitable for intensive induction chemotherapy [10]. Meanwhile, Further trials involving the use of venetoclax in combination therapies are also still being conducted in other malignancies [11].

Due to its high selectivity for the BCL-2 protein, venetoclax shows superior efficacy and better safety profiles compared with other drugs [12,13]. However, the continued use of venetoclax at high doses can result in life-threatening tumor lysis syndrome (TLS, $12 \%)$ and the most frequent adverse events including neutropenia (45\%), diarrhea (35\%), nausea (33\%), anemia (29\%), and thrombocytopenia (22\%) [14]. Furthermore, venetoclax has a high inter-patient' variability with nonlinearity in relative bioavailability $[15,16]$. According to its pharmacokinetics, venetoclax reaches peak plasma concentration 5-8 $\mathrm{h}$ after an oral administration and has a high plasma protein bound rate $(>99 \%)$ with a large apparent volume of distribution [9]. Venetoclax is predominantly metabolized by CYP3A4, also a substrate for OATP1B3, P-glycoprotein (P-gp), and breast cancer-resistance protein transporters (BCRP) [17]. Reports showed that the mean $\mathrm{C}_{\max }$ of venetoclax was $94 \%$ higher in Chinese subjects compared with non-Asian subjects receiving the same dose [18].

Considering the wide interindividual variability in pharmacokinetics and therapeutic response of patients towards anticancer drugs, it is not possible to predict whether an individual patient will reach an adequate plasma exposure using a standard fixed dose of the drug. Therapeutic drug monitoring (TDM) is the measurement of specific drugs ${ }^{\prime}$ stable concentration in a patient's bloodstream, thereby optimizing individual dosage regimens [19]. It is commonly and successfully used to ensure appropriate drug exposure and to limit dose-related toxicities [20]. TDM is cost-effective, and it could be beneficial to personalize a dose to fit the specific needs of cancer patients [21-23]. At present, TDM results concerning venetoclax have few precedents in scientific literature, and there are only a few studies on the quantitation of venetoclax in biological fluids. These methods include liquid scintillation counting (LSC), high-performance liquid chromatography (HPLC), and liquid chromatography tandem mass spectrometry (LC-MS/MS) [24,25]. However, the above methods often require multiple sample preparation steps such as liquid-liquid extraction (LLE), solid phase extraction (SPE), and a long-running program. Choo et al. mentioned a rapid LC-MS/MS method for quantification of venetoclax in dogs' plasma within $1.6 \mathrm{~min}$ [26], but did not present the method validation; thus, the reproducibility of the method, perhaps, is uncertain. Reddy et al. developed a single and accurate LC-MS/MS method to detect venetoclax in rats' plasma, over the range of 5-500 ng/mL under a timeconsuming sample dilution [27]; however, it is unsuitable for TDM to capture inter-intra individual variability in case of overdose. Thus, it is necessary to develop a more sensitive and efficient method to detect venetoclax for high-throughput TDM analysis in humans.

The purpose of the work presented herein was to describe the development and validation of a convenient method for the rapid and reliable quantitation of venetoclax in human plasma. Additionally, the validated method was applied to the therapeutic drug monitoring of Chinese AML patients who were undergoing venetoclax therapy, and whether the peak and trough concentrations of venetoclax are related to the clinical features was explored, which resulted in the large interindividual variation of venetoclax exposure.

\section{Results and Discussion}

\subsection{Method Development and Optimization}

The optimizations of mass spectrum conditions for venetoclax and internal standard(IS) were acquired by continuous infusion of each analyte under the concentration of $500 \mathrm{ng} / \mathrm{mL}$ dissolved in ACN/water (50:50, v/v), via the mass spectrometer fluidic pump at a flow rate of $5 \mu \mathrm{L} / \mathrm{min}$. Considering of the PKa value, the positive electrospray ionization $\left(\mathrm{ESI}^{+}\right)$mode was selected in our method with the two most abundant fragments of $\mathrm{m} / \mathrm{z} 868.5 \rightarrow 321.0,868.5 \rightarrow 636.3$ for venetoclax, and $m / z 875.5 \rightarrow 321.0,875.5 \rightarrow 636.3$ for IS; quantification and confirmation ions were monitored for each analyte. Subsequently, 
cone voltage and collision energy for those transitions were optimized to achieve sufficient MRM signal (Table 1).

Table 1. Relevant LC-MS/MS characteristics. internal standard, IS.

\begin{tabular}{|c|c|c|c|c|c|}
\hline \multirow{2}{*}{ Compound } & \multirow{2}{*}{$\begin{array}{l}\text { Retention Time } \\
\qquad\left(t_{R}, \min \right)\end{array}$} & \multicolumn{2}{|c|}{ Transition $(m / z)$} & \multirow{2}{*}{ Cone Voltage (V) } & \multirow{2}{*}{$\begin{array}{l}\text { Collision } \\
\text { Energy (V) }\end{array}$} \\
\hline & & Quantification & Confirmation & & \\
\hline venetoclax & 1.8 & $868.5 \rightarrow 321.0$ & $868.5 \rightarrow 636.3$ & 70 & 36 \\
\hline IS & 1.8 & $875.5 \rightarrow 321.0$ & $875.5 \rightarrow 636.3$ & 60 & 40 \\
\hline
\end{tabular}

Internal standard, IS.

The composition of the mobile phase directly affects the separation and ionization. Due to the pKa and $\log P$ values, the mobile phase comprised ACN and water containing $0.1 \%$ formic acid was chosen to improve chromatographic performance in our method. The total procedure, including washing and re-equilibrating steps, took $4 \mathrm{~min}$, shorter than the time spent in previously published method. Although Choo et al. provided a faster separation method in only $1.6 \mathrm{~min}$ [26], the detail of gradient elution was not presented, and whether it contained the washing and equilibrating procedure is unknown. Without a washing procedure, the remaining salt or other plasma components may affect column performance and MS ionization. In addition, a limitation of the work of Choo et al. is lacking the comprehensive method validation. Furthermore, the composition of the mobile phase in our assay is more convenient than the method published by Choo et al. and Reddy et al., which added $2 \mathrm{mM}$ ammonium acetate in water and acetonitrile with $0.1 \%$ formic acid $[26,27]$. Some other methods have been reported with a time-consuming and complicated SPE, and LLE for sample pretreatment [24,25]. However, the sample preparation strategy selected in our experiment was $\mathrm{ACN}$ protein precipitation with only single-step extraction, evaluated by matrix effect for three concentrations, which is a fast, cost-effective, and manageable procedure for routine measurements in clinical settings. In addition, the quantitative methods created by Choo et al. and Reddy et al. were used in determination of dog or rat plasma, rather than human plasma analysis [26,27]. Here, we provided the summary of analytical methods for quantification of venetoclax (Table S1, Supplementary Materials).

In our study, we described an advantageous analysis to determine venetoclax in human plasma over the published multiple-analyte method, which is characterized by a more laborious sample preparation procedure (e.g., liquid-liquid extraction) and a complex mobile phase (e.g., ammonium aqueous solution or $\mathrm{ACN}-\mathrm{MeOH}, 50 / 50 \mathrm{v} / \mathrm{v}$ ); meanwhile, our method seems more suitable for clinical high-throughput TDM analysis with a good sensitivity in a short time.

\subsection{Method Validation}

\subsubsection{Linearity, Selectivity and Specificity}

Typical MRM chromatograms of blank plasma, blank matrix spiked with IS, and venetoclax at a lower limit of quantification (LLOQ), as well as a sample from an AML patient during venetoclax treatment are presented in Figure 1. The retention time of venetoclax and IS were 1.8 and $1.8 \mathrm{~min}$, respectively. No significant interfering peaks were observed around the retention time of venetoclax and IS, illustrating that the method was specific for the analyte determination. 


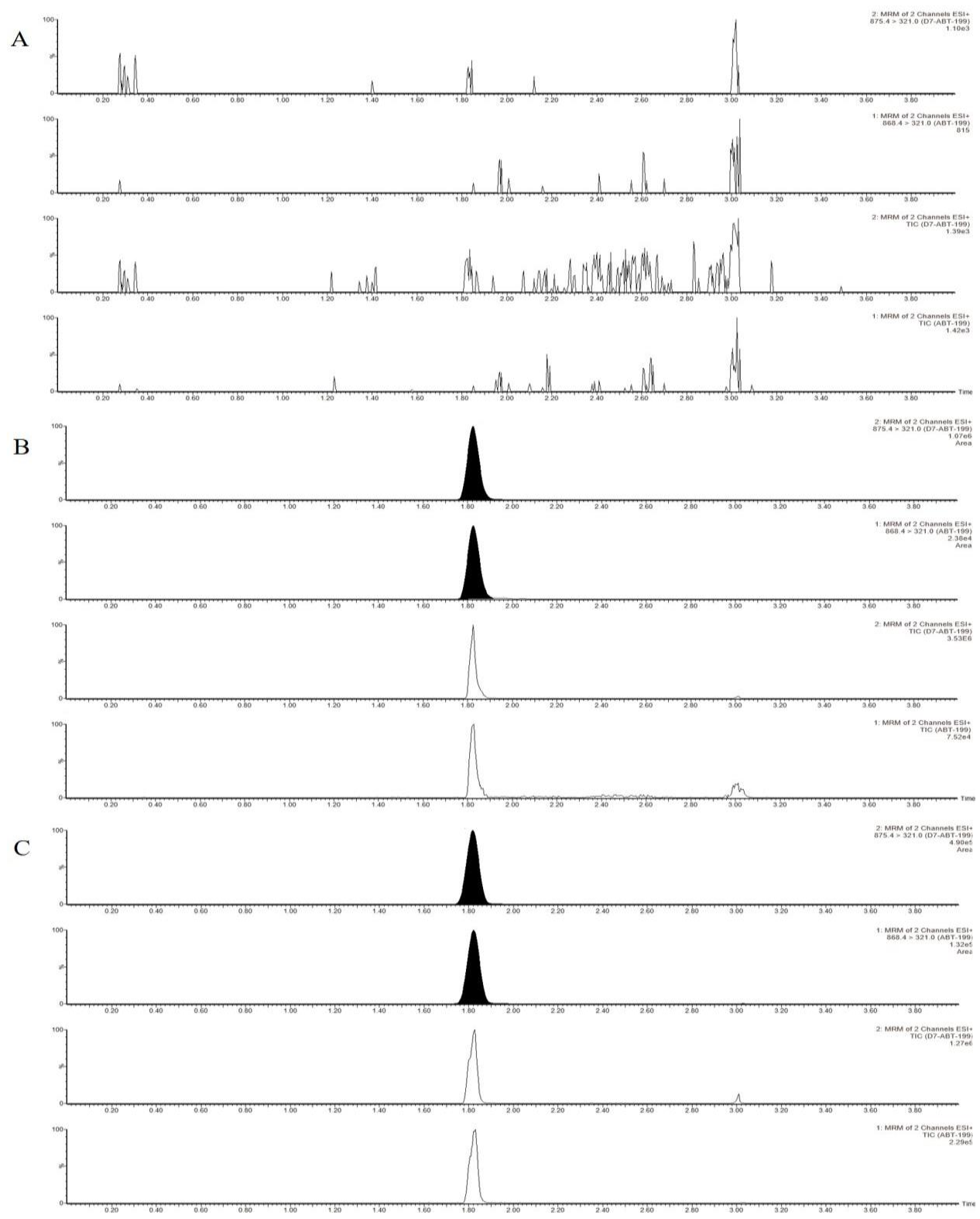

Figure 1. Representative UPLC-MS/MS chromatograms of venetoclax and IS (A)_blank plasma; (B) —blank plasma-spiking venetoclax and IS; (C)—plasma sample $0.5 \mathrm{~h}$ before continuous oral administration of venetoclax at steady state).

The calibration curve of venetoclax was validated at seven points over the concentration range of $25-8000 \mathrm{ng} / \mathrm{mL}$ covering the plasma therapeutic range with a coefficient of correlation $\left(\mathrm{r}^{2} \geq 0.997\right.$, Figure 2), following the regression equation $(y=0.0003816 x+0.05653)$. Each concentration was analyzed in three replicates and the precision for each calibrator with RSD ranged from $0.38 \%$ to $5.35 \%$. According to several literatures, the plasma concentration of venetoclax varied from 30 to $4000 \mathrm{ng} / \mathrm{mL}$ in human (including healthy volunteers, chronic lymphocytic leukemia patients, and small lymphocytic lymphoma patients) across the dose levels (100-600 mg) [28-31], indicating that the calibration curve is capable of measuring the concentration of venetoclax in human plasma by encompassing both peak and trough concentrations. 


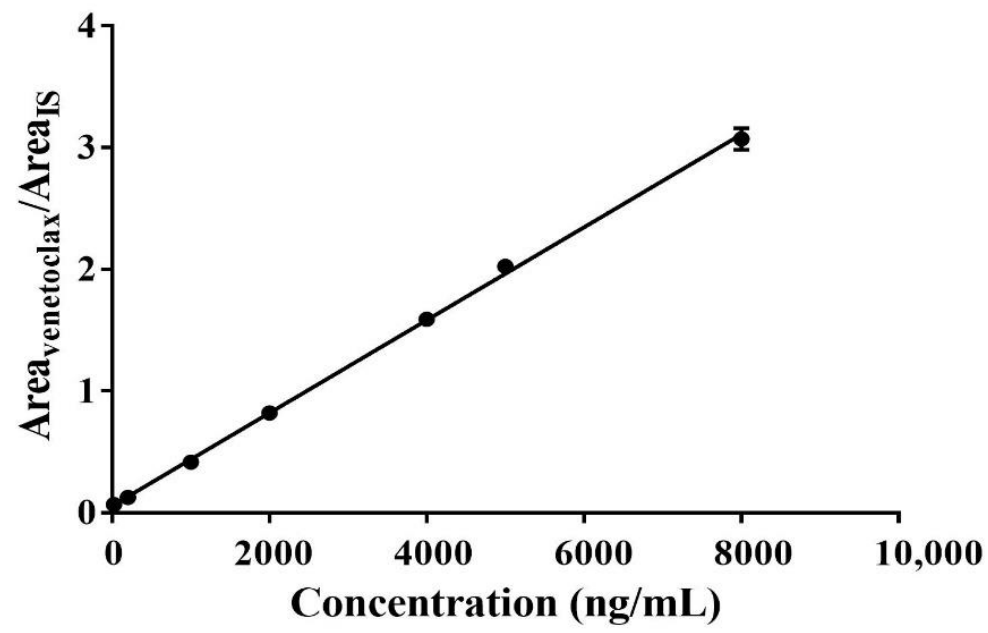

Figure 2. The linear relationship between the analyte concentration and the peak area ratios $(n=3)$.

\subsubsection{Precision and Accuracy}

Results concerning precision and accuracy over low-concentration quality control (LQC), middle-concentration quality control (MQC), high-concentration quality control (HQC), and lower limit of quantification (LLOQ) samples are presented in Table 2. The intra-day and inter-day precision (relative standard deviation (\%RSD)) for venetoclax ranged from $1.99 \%$ to $6.18 \%$ and $4.13 \%$ to $7.30 \%$. Furthermore, the intra- and inter-day accuracy (relative error (\%RE)) varied from $-6.51 \%$ to $0.95 \%$ and $-4.81 \%$ to $0.49 \%$, respectively. Therefore, the results of precision and accuracy met the acceptable criteria of FDA guidelines.

Table 2. Intra-and inter-day precision and accuracy of venetoclax (mean $\pm \mathrm{SD}, n=6$ ).

\begin{tabular}{cccccccc}
\hline Compound & $\begin{array}{c}\text { Concentration } \\
\text { Spiked } \\
\text { (ng/mL) }\end{array}$ & $\begin{array}{c}\text { Intra-Day } \\
\text { Concentration } \\
\text { Measured (ng/mL) }\end{array}$ & $\begin{array}{c}\text { Accuracy } \\
\text { RE (\%) }\end{array}$ & $\begin{array}{c}\text { Precision } \\
\text { RSD (\%) }\end{array}$ & $\begin{array}{c}\text { Inter-Day } \\
\text { Concentration } \\
\text { Measured (ng/mL) }\end{array}$ & $\begin{array}{c}\text { Accuracy } \\
\text { RE (\%) }\end{array}$ & $\begin{array}{c}\text { Precision } \\
\text { RSD (\%) }\end{array}$ \\
\hline \multirow{3}{*}{ Venetoclax } & 25 & $24.80 \pm 0.57$ & -0.80 & 2.28 & $25.12 \pm 1.64$ & 0.49 & 6.51 \\
& 50 & $51.35 \pm 3.17$ & 2.70 & 6.18 & $48.44 \pm 3.54$ & -3.11 & 7.30 \\
& 2500 & $2523.77 \pm 89.95$ & 0.95 & 3.56 & $2419.72 \pm 122.66$ & -3.21 & 5.07 \\
\hline
\end{tabular}

\subsubsection{Recovery and Matrix Effect}

Results of extraction recovery and matrix effect at three level of QCs concentrations are depicted in Table 3. The extraction recovery of venetoclax in human plasma was nearly $100 \%$ with an RSD $\leq 5.37 \%$, while the extraction recovery of IS was $104.41 \pm 2.76 \%$. Matrix effects of the analyte was in the range of $84.5-95.2 \%$ with RSD $\leq 5.33 \%$, and the IS matrix effect was $90.19 \% \pm 2.34 \%$. This demonstrated that the developed method had a highly efficient extraction and the matrix effect was concentration-independent.

Table 3. Extraction recovery and matrix effect of venetoclax and IS (mean $\pm \mathrm{SD}, n=6$ ).

\begin{tabular}{cccccc}
\hline \multirow{2}{*}{ Compound } & \multirow{2}{*}{$\begin{array}{c}\text { Concentration } \\
\text { Spiked (ng/mL) }\end{array}$} & Mean \pm SD & RSD\% & \multicolumn{2}{c}{ Matrix Effect (\%) } \\
\cline { 3 - 6 } & 50 & $108.36 \pm 2.63$ & 2.63 & $89.77 \pm 4.78$ & 5.33 \\
\hline Venetoclax & 2500 & $106.24 \pm 1.93$ & 1.82 & $89.36 \pm 3.38$ & 3.78 \\
& 6000 & $106.64 \pm 5.73$ & 5.37 & $91.12 \pm 2.02$ & 2.21 \\
IS & 1000 & $104.41 \pm 2.76$ & 2.62 & $90.19 \pm 2.34$ & 2.60 \\
\hline
\end{tabular}




\subsubsection{Carry-Over}

Carryover was evaluated by the detection of blank sample following upper limit of quantification (ULOQ) samples, and there was no response at the corresponding retention times, indicating no carryover occurred.

\subsubsection{Stability}

The results of stability at two QC concentration levels in plasma under a variety of conditions are summarized in Table 4 . The accuracy (\%RE) and precision (\%RSD) were in the range of $-8.66-0.76 \%$ and $1.0-4.6 \%$, respectively. The results demonstrated a good stability of venetoclax throughout the experiment.

Table 4. Stability of venetoclax in plasma under different conditions. (mean $\pm \mathrm{SD}, n=3$ ).

\begin{tabular}{|c|c|c|c|c|}
\hline $\begin{array}{l}\text { Spiked } \\
(\mathrm{ng} / \mathrm{mL})\end{array}$ & Condition & $\begin{array}{c}\text { Found } \\
(\mathrm{ng} / \mathrm{mL}, \text { Mean } \pm \mathrm{SD})\end{array}$ & $\begin{array}{l}\text { Precision } \\
\text { RSD (\%) }\end{array}$ & $\begin{array}{c}\text { Accuracy } \\
\text { RE (\%) }\end{array}$ \\
\hline \multirow{4}{*}{50} & Room temperature for $6 \mathrm{~h}$ & $45.67 \pm 1.12$ & 2.4 & -8.66 \\
\hline & $-80^{\circ} \mathrm{C}$ for 31 days & $49.57 \pm 1.15$ & 2.3 & -0.87 \\
\hline & Three freeze-thaw cycles & $48.77 \pm 1.53$ & 3.1 & -5.38 \\
\hline & Autosampler in $4{ }^{\circ} \mathrm{C}$ for $8 \mathrm{~h}$ & $46.70 \pm 2.13$ & 4.6 & -6.60 \\
\hline \multirow{4}{*}{6000} & Room temperature for $6 \mathrm{~h}$ & $6045.47 \pm 152.82$ & 2.5 & 0.76 \\
\hline & $-80{ }^{\circ} \mathrm{C}$ for 31 days & $5618.17 \pm 56.22$ & 1.0 & -6.36 \\
\hline & Three freeze-thaw cycles & $5676.83 \pm 71.15$ & 1.3 & -2.5 \\
\hline & Autosampler in $4^{\circ} \mathrm{C}$ for $8 \mathrm{~h}$ & $5800.63 \pm 168.44$ & 2.9 & -3.32 \\
\hline
\end{tabular}

\subsection{Patient Characteristics and Venetoclax Concentrations}

The analysis included 109 venetoclax plasma concentrations from 62 AML patients. Their demographic and clinical characteristics are displayed in Table 5. Patients' median age was 62 years (range, 18-90), and 36/62 (58.1\%) were male.

Table 5. Patients' demographic data and clinical characteristics. $(n=62)$.

\begin{tabular}{ccc}
\hline Characteristic & Mean $( \pm \mathbf{S D})$ & Median (Range) \\
\hline Age $($ year $)$ & $58.9 \pm 14.3$ & $62(18.0-90.0)$ \\
Height $(\mathrm{cm})$ & $165.5 \pm 5.5$ & $165.0(153.0-177.0)$ \\
Weight $(\mathrm{kg})$ & $63.44 \pm 10.48$ & $62.25(43.5-92.0)$ \\
BMI $\left(\mathrm{kg} / \mathrm{m}^{2}\right)$ & $23.1 \pm 3.3$ & $23.0(16.9-31.46)$ \\
Gender $($ Female/Male) & $26 / 36$ & \\
ALB $(\mathrm{g} / \mathrm{L})$ & $40.1 \pm 5.65$ & $40.85(25.7-51)$ \\
ALT $(\mathrm{U} / \mathrm{L})$ & $15.08 \pm 8.65$ & $14(3-54)$ \\
AST $(\mathrm{U} / \mathrm{L})$ & $20.45 \pm 10.66$ & $17(4-62)$ \\
BUN $(\mathrm{mmol} / \mathrm{L})$ & $5.83 \pm 2.61$ & $5.41(1.94-18.70)$ \\
CREA $(\mu \mathrm{mol} / \mathrm{L})$ & $77.4 \pm 30.6$ & $69.5(42.0-224.0)$ \\
$\mathrm{LDH}(\mathrm{U} / \mathrm{L})$ & $358 \pm 325.1$ & $234.5(54-2066)$ \\
WBC $\left(10^{\wedge} / \mathrm{L}\right)$ & $9.37 \pm 14.34$ & $3.94(0.77-83.59)$ \\
NEUT $\left(10^{\wedge} 9 / \mathrm{L}\right)$ & $2.60 \pm 5.21$ & $1.485(0.06-31.29)$ \\
$\mathrm{HGB}(\mathrm{g} / \mathrm{L})$ & $86.4 \pm 24.85$ & $83(45-137)$ \\
PLT $\left(10^{\wedge} / \mathrm{L}\right)$ & $85.18 \pm 151.9$ & $33.5(3-1097)$ \\
$\mathrm{C}_{\max }(\mathrm{ng} / \mathrm{mL}, n=61)$ & $2966.0 \pm 1595.0$ & $2408.0(515.4-7189.0)$ \\
$\mathrm{C}_{\min }(\mathrm{ng} / \mathrm{mL}, n=48)$. & $1018.0 \pm 729.4$ & $836.6(74.2-3257.0)$
\end{tabular}

ALB, albumin; ALT, alanine aminotransferase; AST, aspartate transaminase; BUN, blood urea; CREA, creatinine; LDH, lactate dehydrogenase; WBC, white blood cell count; NEUT, neutrophil; HGB, hemoglobin; PLT, platelet.

The developed UPLC-MS/MS method was applied to measure venetoclax concentrations in plasma. The peak plasma concentrations $\left(C_{\max }\right)$ of venetoclax in Chinese AML patient were $2966.0 \pm 1595.0 \mathrm{ng} / \mathrm{mL}$, while the trough concentrations $\left(C_{\min }\right)$ were 
$1018.0 \pm 729.4 \mathrm{ng} / \mathrm{mL}$ (Figure 3). Both $\mathrm{C}_{\max }$ and $\mathrm{C}_{\min }$ exhibited a large interindividual variability, and this observation is consistent with previous studies.
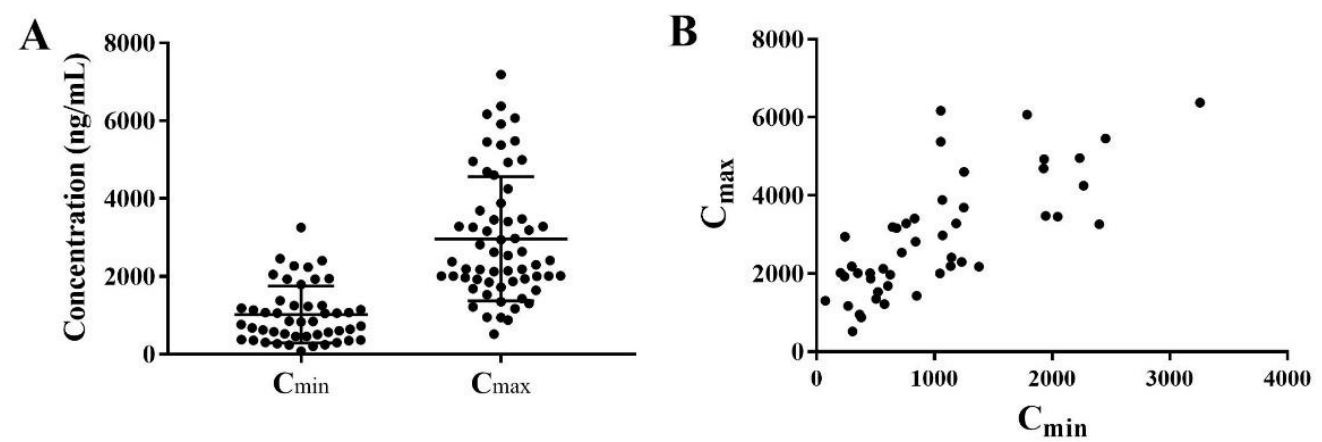

Figure 3. Distribution of venetoclax trough concentrations and peak concentrations in Chinese AML patients (A)-summary of venetoclax concentrations, (B) - paired of $C_{\min }$ and $C_{\max }$ ).

\subsection{Relationship between Covariates and Venetoclax Concentrations}

The $\mathrm{C}_{\min }$ showed no difference between the female cohort and male cohort. Similarly, no correlation was observed between sex and $C_{\max }$ (Figure $4 \mathrm{~A}$ ), in line with the previous study performed by Deng et al. [32]. In the literature, venetoclax's volume of distribution is $30 \%$ lower in females than in males, and this difference in apparent volume is not considered clinically relevant with $\mathrm{C}_{\max }$ [33]. Correlation heatmap displayed the relationship between concentrations and the characteristics (Figure 4B). Red color indicates a positive correlation and blue color indicates a negative correlation. In addition, a significant correlation was observed between aspartate transaminase (AST) levels and venetoclax concentrations $\left(C_{\min }\right.$ and $\left.C_{\max }\right)$, and AST showed a clinically relevant effect on $C_{\min }$ and $C_{\max }(p<0.01, \mathrm{r}>0.3$, Figure 4C-D). Moreover, alanine aminotransferase (ALT) showed a relevant effect on $C_{\min }$ $(p<0.01, r>0.3$, Figure $4 \mathrm{E})$, but it had no clinically relevant effect on $C_{\max }(p>0.01, r<0.3$, Figure 4E). However, Deng et al. did not find any effect on clearance with AST or ALT levels and estimated venetoclax in linear elimination [32]. Apart from the individual differences and insufficient sample size in our experiment, the divergence may due to the effects of ethnicity and type of leukemia on pharmacokinetics; only $1.19 \%$ of patients enrolled were Asian (1.19\%) with CLL in Deng et al. [32], while our experiment was carried out in Chinese AML patients. Besides, there was no change of AST and ALT level after treatment. The comparison of AST and ALT levels before and after treatment is displayed in the Supplementary Materials (Figure S1). According to its pharmacokinetic process, venetoclax is predominantly eliminated by the liver. In view of USA National Cancer Institute (NCI) Organ Dysfunction Working Group for Hepatic Dysfunction, AST levels and ALT levels are used as indicators for evaluating liver function [34]. At the same time, it is noted that venetoclax dosage adjustment is required in subjects with severe hepatic impairment in another study, which recommended a 50\% dose reduction of venetoclax [35]. It is important to monitor the concentration in hepatic impairment patients during venetoclax therapy. 
A

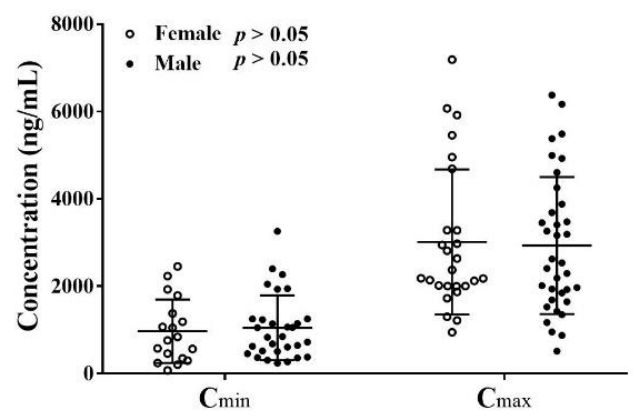

C

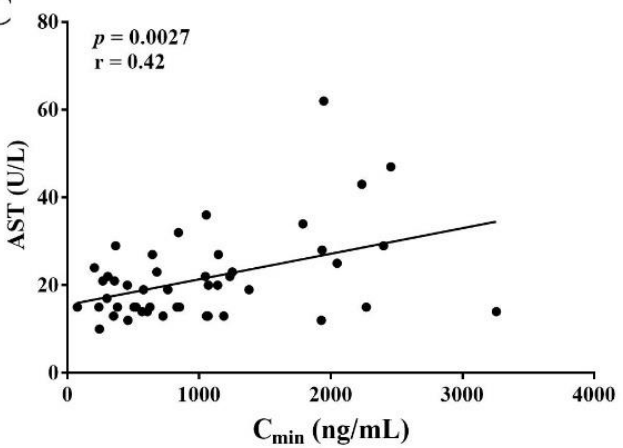

E

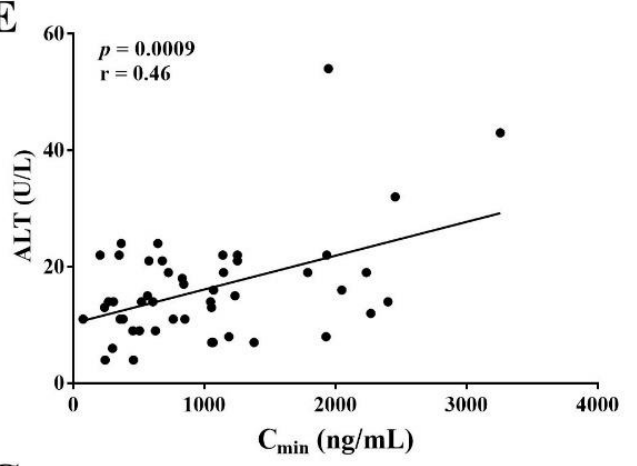

G

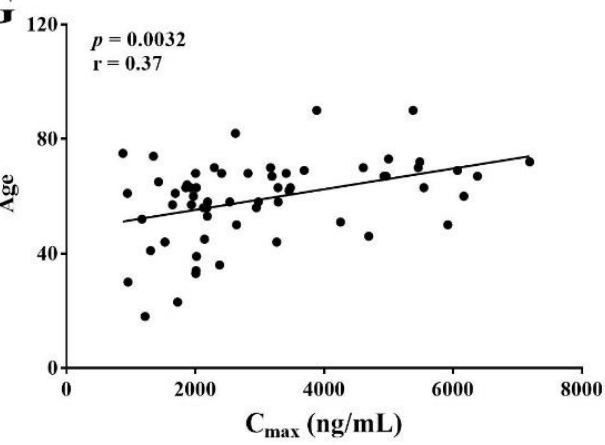

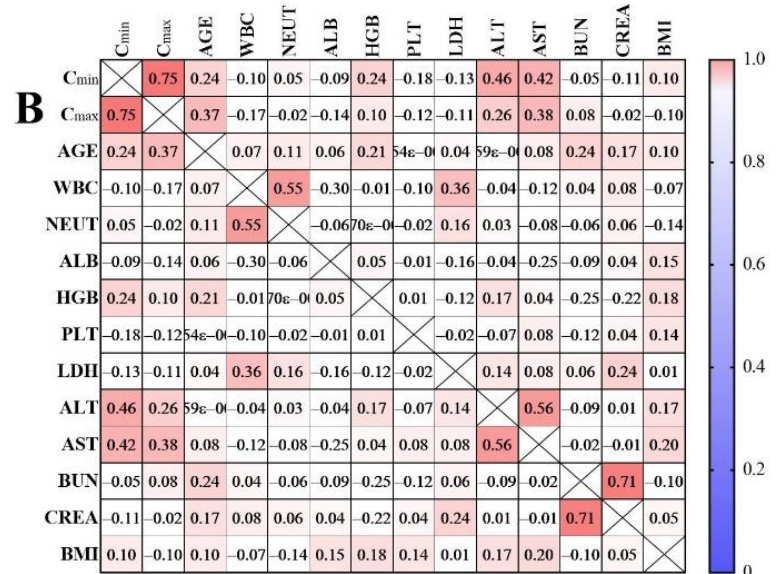

D

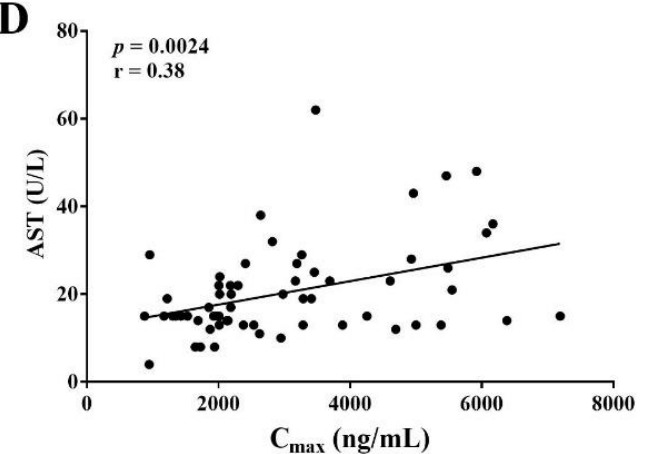

F

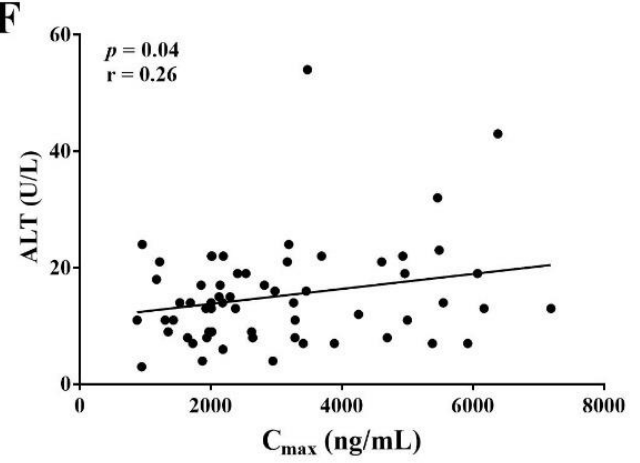

Figure 4. Variables associated with the venetoclax concentrations. The venetoclax concentrations in the female cohort were similar to the male cohort (A). The correlation heatmap between venetoclax concentrations and clinical features (B). The AST concentration and the venetoclax concentration were positively associated (C,D); The ALT showed a relevant effect on $C_{\min }$ rather than $C_{\max }(\mathbf{E}, \mathbf{F})$; The age and the $C_{\max }$ of venetoclax were positively associated $(G)$. WBC, white blood cell count; NEUT, neutrophil; HGB, hemoglobin; ALB, albumin; PLT, platelet; LDH, lactate dehydrogenase; ALT, alanine aminotransferase; AST, aspartate transaminase; BUN, blood urea; CREA, creatinine. 
Meanwhile, a markedly raised venetoclax peak concentration was associated with the growth of age $(p<0.01$, Figure $4 \mathrm{E})$, partially as a result of the decreased activity of intestine-CYP3A4 and P-gp in elderly patients. As apparent volume of distribution of venetoclax is very large (241 L) [9], a variation in albumin will have a rare impact on the variation in volume of distribution in spite of the large protein-bound rate. Consequently, a negligible influence on $C_{\max }$ and $C_{\min }$ by albumin was observed $(p>0.05 ; r=-0.09$, $-0.14)$.

Several factors may influence the systemic exposure in patients, including variability in oral drug absorption, distribution, metabolism, and elimination. Additionally, patient characteristics such as age, sex, body weight, renal function, and liver function also contribute to variability in plasma exposure. TDM is the quantification of real-time drug concentrations in blood, and it considers the interindividual variability of pharmacokinetics and enables personalized pharmacotherapy. It is now widely used in many areas of clinical medicine, such as antibiotics, antipsychotic drugs, and immunosuppressants, but its use for anticancer therapies has been limited because of the undefined concentrationeffect relationships. Nonetheless, targeted anticancer drugs show a large inter-individual pharmacokinetic (PK) variability and a narrow therapeutic index, which fit many criteria defined as prerequisites for utilizing TDM approaches. TDM has been used to improve the clinical use of chemotherapy agents partly, for example, methotrexate, imatinib, and so on. However, further clinical research evaluating the impact of TDM on overall survival or other pharmacodynamic outcomes needs to be carried out. In the current study, we observed a high inter-individual variability in both $C_{\max }$ and $C_{\min }$, but the venetoclax peak concentrations were significantly higher in AML patients with AST levels increasing. ALT showed a relevant effect on $C_{\min }$ instead of $C_{\max }$. AST and ALT levels are predictive indexes of disease and can reflect liver function. Deng et al. and Salem et al. showed that severe hepatic impairment and a stronger inhibitor of CYP3A4, OATP1B3, have an impact on the venetoclax clearance parameter $[32,35]$, so it seems that liver function may be a key factor in inter-individual variability. Besides, intestinal CYP3A4 and P-gp mediated the first-pass metabolism and provided a basis for inter-individual differences in oral absorption while hepatic CYP3A4, OATP1B3 was involved in drug elimination and offered a theory for inter-individual differences in clearance. Thus, the influence of genetic variants on venetoclax concentration needs to be further studied.

\section{Material and Methods}

\subsection{Chemicals and Reagents}

Venetoclax (ABT-199, purity $\geq 98.5 \%$ ) was purchased from GLPBIO, $\left[{ }^{2} \mathrm{H}_{7}\right]$-venetoclax (D7-ABT-199, purity $\geq 98.5 \%$ ) was provided by Alsachim, used as an internal standard, and Acetonitrile (ACN) was supplied by Merck\&Co., Inc. (Darmstadt, Germany). Ultrapure water was acquired from a Milli- $\mathrm{Q}^{\circledR}$ Water Purification System (Millipore-lbérica, Madrid, Spain). Dimethyl sulfoxide (DMSO) and formic acid were purchased from Sigma-Aldrich (Madrid, Spain). Solvents were all of LC-MS grade. Blank human plasma was obtained from RILD Co. Ltd. (Shanghai, China).

\subsection{Calibrators and QCS}

Stock solutions of venetoclax and $\left[{ }^{2} \mathrm{H}_{7}\right]$-venetoclax were prepared in DMSO at concentrations of $2 \mathrm{mg} / \mathrm{mL}$. The IS working solution consisted of $1 \mu \mathrm{g} / \mathrm{mL}\left[{ }^{2} \mathrm{H}_{7}\right]$-venetoclax in ACN. Calibrators were prepared in DMSO at seven concentrations, based on the clinical ranges, and spiked into blank plasma samples at the concentration of 25, 200, 1000, 2000, 4000,5000 , and $8000 \mathrm{ng} / \mathrm{mL}$. The QC samples for method validation at LLOQ $(25 \mathrm{ng} / \mathrm{mL})$, low $(50 \mathrm{ng} / \mathrm{mL})$, medium $(2500 \mathrm{ng} / \mathrm{mL})$, and high $(6000 \mathrm{ng} / \mathrm{mL})$ were also prepared by the above method. The amount of solvent added to the matrix, both for calibrators and QCs, did not exceed $2 \%$. 


\subsection{Sample Preparation}

Plasma samples were subjected to protein precipitation, and an aliquot of $100 \mu \mathrm{L}$ sample was prepared in an Eppendorf tube. Subsequently, $300 \mu \mathrm{L}$ of IS working solution in ACN was added, followed by vertexing for $3 \mathrm{~min}$ and centrifugation at 13,000 rpm for $10 \mathrm{~min} ; 100 \mu \mathrm{L}$ of the supernatant was transferred to HPLC vial and $2 \mu \mathrm{L}$ was injected onto the UPLC ${ }^{\circledR}$ column.

\subsection{UPLC-MS/MS Conditions}

The UPLC-MS/MS system was comprised of an ACQUITY UPLC I-Class separation module (Waters, Milford, USA) coupled to a Xevo TQ-S micro triple-quadrupole mass spectrometer (Waters, Milford, CT, USA) in positive mode, and Chromatographic separation was conducted on an ACQUITY UPLC BEH C18 column $(2.1 \times 100 \mathrm{~mm}, 1.8 \mu \mathrm{m}$, Waters) equipped with an in-line filter $(0.2 \mu \mathrm{m})$, maintained at $35{ }^{\circ} \mathrm{C}$ with a gradient elution (0-0.3 $\mathrm{min}, 5 \%$ of B; $0.3-2.0 \mathrm{~min}, 5-95 \%$ of B; $2.0-2.5 \mathrm{~min}, 95 \%$ of B, 2.5-2.6 $\mathrm{min}, 95 \%-5 \%$ of $\mathrm{B}, 2.6-4.0 \mathrm{~min}, 5 \%$ of $\mathrm{B}$ ) at $0.4 \mathrm{~mL} \mathrm{~min}^{-1}$, where mobile phase $\mathrm{A}$ and $\mathrm{B}$ were $0.1 \%$ formic acid in water and acetonitrile (ACN), respectively. The autosampler temperature was set at $4{ }^{\circ} \mathrm{C}$. Under these conditions, venetoclax and $\left[{ }^{2} \mathrm{H}_{7}\right]$-venetoclax (IS) typically eluted at 1.8 and $1.8 \mathrm{~min}$.

Analytes were quantified in multiple-reaction monitoring (MRM) mode at $m / z$ transitions of $868.5 \rightarrow 321.0,868.5 \rightarrow 636.3$ for venetoclax and $875.5 \rightarrow 321.0,876.5 \rightarrow 636.3$ for $\left[{ }^{2} \mathrm{H}_{7}\right]$-venetoclax (IS), respectively. The source temperature was set at $150{ }^{\circ} \mathrm{C}$ and the ion spray voltage at $3.0 \mathrm{kV}$, nitrogen was used as nebulizing $\left(50 \mathrm{~L} \cdot \mathrm{Hr}^{-1}\right)$ and drying gas $\left(600 \mathrm{~L} \cdot \mathrm{Hr}^{-1}\right)$ at $500{ }^{\circ} \mathrm{C}$, and the fragmentor voltage (volt, $\left.\mathrm{V}\right)$ and Collision energy $(\mathrm{CE}, \mathrm{eV})$ were optimized and set at $70 \mathrm{~V}, 36 \mathrm{~V} ; 60 \mathrm{~V}, 40 \mathrm{~V}$ for venetoclax and IS, respectively. Data acquisition and processing were controlled by Waters MassLynx software (version 4.2).

\subsection{Method Validation}

The validation of this method was based on FDA guidelines (FDA, Guidance for Industry Bioanalytical Method Validation, 2018.) [36] including calibration curve, precision, accuracy, selectivity, matrix effect, recovery, carry-over, and stability.

\subsubsection{Calibration Curve, Selectivity and Specificity}

The calibration curve was generated by plotting peak area ratios (analyte/IS) versus the nominal concentration using 7 levels of non-zero standards and a linearly weighed $\left(1 / x^{2}\right)$ least-squares regression model. The error of accuracy and relative standard deviation (RSD, $\%$ ) should be within $15 \%$ of the nominal value for each CAL $(n=3)$ or within $20 \%$ for the lower limit of quantitation (LLOQ, $n=6$ ) with a signal-to-noise ratio $(\mathrm{S} / \mathrm{N}) \geq 10$ for specificity. The selectivity was confirmed by analyzing spiked blank matrix (commercial blank human plasma) at the LLOQ, and the chromatographic peak response compared with the blank matrix should be less than $20 \%$ for the analyte and less than $5 \%$ for the IS.

\subsubsection{Precision and Accuracy}

The intra-day or inter-day precision and accuracy of the method were determined by analyzing the four QC levels (LLOQ: $25 \mathrm{ng} / \mathrm{mL}, \mathrm{LQC}: 50 \mathrm{ng} / \mathrm{mL}, \mathrm{MQC}: 2500 \mathrm{ng} / \mathrm{mL}$ and HQC: $6000 \mathrm{ng} / \mathrm{mL}$ ) in plasma by six replicates on the same day or different three days, respectively. The precision was evaluated as the mean concentration $(\mathrm{ng} / \mathrm{mL}) \pm \mathrm{SD}$ and relative standard deviation (\%RSD) accuracy was expressed as relative error (\%RE), while the value should not exceed $15 \%$, except for LLOQ (RSD $<20 \%$ ).

\subsubsection{Matrix Effect and Recovery}

The extraction recovery was determined at three levels $(50,2500$, and $6000 \mathrm{ng} / \mathrm{mL})$ by comparing the peak area of MER (samples spiked analyte before blank matrix extraction), MEX (samples spiked analyte after blank matrix extraction) and MEP (reconstitution solvent containing equal concentration). 
The equations for evaluation of the extraction recovery (Equation (1)) and matrix effect (Equation (2)) are listed below:

$$
\begin{gathered}
\text { Extraction recovery }=\text { MER } / \text { MEX } \times 100 \% \\
\text { Matrix effect }=\text { MEX } / \text { MEP } \times 100 \%
\end{gathered}
$$

The RE obtained for the IS-normalized matrix factor should be less than $15 \%$.

\subsubsection{Carry-Over}

The blank sample (blank matrix) was injected following the upper limit of quantification (ULOQ, $8000 \mathrm{ng} / \mathrm{mL}$ ) to understand the carry-over effect. The peak area of the analyte and the IS in the blank sample should not exceed $20 \%$ of that obtained for the LLOQ and $5 \%$ of the IS, respectively.

\subsubsection{Stability}

Storage stability was evaluated at low and high QC levels $(50,6000 \mathrm{ng} / \mathrm{mL})$ in three replicates, such as keeping at room temperature for $6 \mathrm{~h},-80{ }^{\circ} \mathrm{C}$ for 31 days, three freeze/thaw cycles of unextracted samples, and the postpreparative stability of samples in an autosampler at $4{ }^{\circ} \mathrm{C}$ for $8 \mathrm{~h}$ was also tested. Samples were considered stable if the ratio between original concentration and concentration under different storages was less than $15 \%$.

\subsection{Application}

The method was successfully applied to determine the stable trough plasma concentration $\left(C_{\min }\right)$ and peak plasma concentration $\left(C_{\max }\right)$ of 109 plasma samples from 62 AML patients. Briefly, patients received a daily dose escalation of venetoclax of $100 \mathrm{mg}$ (Day1), $200 \mathrm{mg}$ (Day2), to a final dose of $400 \mathrm{mg}$ (Day3) in 28-day cycles with azacytidine $75 \mathrm{mg} / \mathrm{m}^{2} /$ day for 7 days. Considering the $C_{\max }$ may influenced by fasting and dietary intake, venetoclax was taken after a standardized diet (low-fat meal) within $30 \mathrm{~min}$. No patients were administered with CYP3A4, OATP1B3, P-gp inducers/inhibitors. Blood samples were collected in $5 \mathrm{~mL}$ ethylenediaminetetraacetic acid potassium salt (EDTA-K2) tubes on day 10 at steady state, just before the next administration $\left(C_{\min }\right)$ and $6 \mathrm{~h}$ after drug administration $\left(\mathrm{C}_{\max }\right)$. The blood was centrifuged at $4000 \mathrm{~g}$ for $10 \mathrm{~min}$ to separate the plasma and then stored in a $-80{ }^{\circ} \mathrm{C}$ fridge before analysis. The clinical study was approved by the Ethics Committee of first Affiliated Hospital, Zhejiang University School of Medicine (IIT20210153B). All patients provided written informed consent to participate in our study.

\subsection{Statistical Analysis}

Categorical variables were presented as absolute counts. Continuous data were presented as the mean ( \pm standard deviation (SD)) and median (range), and categorical and continuous variables were compared with the Fisher's exact and a two-tailed Student's t-test, respectively. Continuous variables were tested for normality using the KolmogorovSmirnov test. Correlations were assessed using Pearson's or Spearman's coefficient tests. $p$-values $<0.05$ were considered statistically significant and a confidence level of 0.95 was used for estimating intervals. All analyses were conducted using SPSS Statistics version 18.0 (IBM Corp., Armonk, NY, USA) and GraphPad Prism version 7.0 (GraphPad Software, La Jolla, CA, USA).

\section{Conclusions}

We described a specific, sensitive, and rapid UPLC-MS/MS method for quantification of venetoclax in human plasma. The method was successfully validated and applied to patient samples with a good performance in selectivity, linearity, precision, accuracy, and stability. In our study, a wide variability in venetoclax concentrations was observed, consistent with the results of previous studies. More importantly, $C_{\max }$ and $C_{\min }$ of venetoclax 
showed a positive correlation with AST levels, but ALT had no clinically relevant effect on $\mathrm{C}_{\max }$. At the same time, $\mathrm{C}_{\max }$ of venetoclax was significantly higher in the older patients. This makes it necessary to consider TDM could be a useful tool in patients undergoing venetoclax treatment, especially with hepatic impairment.

Supplementary Materials: The following are available online, Figure S1: The comparison of AST (A) and ALT (B) levels before and after treatment, Table S1: Summary of analytical performance for quantification of Venetoclax.

Author Contributions: Data curation, X.Y. and C.M.; formal analysis, X.Y. and X.H.; investigation, L.H.; methodology, X.Y.; project administration, X.Y.; resources, H.T.; software, X.Y.; supervision, X.L., H.T. and Y.L.; validation, X.H.; writing—original draft, X.Y.; writing-review and editing, Y.L. All authors have read and agreed to the published version of the manuscript.

Funding: This research received no external funding. We were especially grateful for the patients' cooperation.

Institutional Review Board Statement: The clinical study was approved by the Ethics Committee of first Affiliated Hospital, Zhejiang University School of Medicine (IIT20210153B).

Informed Consent Statement: All patients were provided written informed consent to participate in our study.

Data Availability Statement: All data included in this study are available upon request by contact with the corresponding author.

Acknowledgments: We thank the associate editor and the reviewers for their useful feedback that improved this paper.

Conflicts of Interest: Research data are not shared; the authors have no conflict of interest to declare.

Sample Availability: Samples of the compounds are not available from the authors.

\section{References}

1. Stirewalt, D.L.; Radich, J.P. The role of FLT3 in haematopoietic malignancies. Nat. Rev. Cancer 2003, 3, 650-665. [CrossRef] [PubMed]

2. Woyach, J.A.; Johnson, A.J. Targeted therapies in CLL: Mechanisms of resistance and strategies for management. Blood 2015, 126, 471-477. [CrossRef] [PubMed]

3. Papanicolas, L.E.; Gordon, D.L.; Wesselingh, S.L.; Rogers, G.B. Not Just Antibiotics: Is Cancer Chemotherapy Driving Antimicrobial Resistance? Trends Microbiol. 2018, 26, 393-400. [CrossRef] [PubMed]

4. Campbell, K.J.; Tait, S.W.G. Targeting BCL-2 regulated apoptosis in cancer. Open Biol. 2018, 26, 393-400. [CrossRef]

5. Dai, H.; Ding, H.; Meng, X.W.; Lee, S.H.; Schneider, P.A.; Kaufmann, S.H. Contribution of Bcl-2 phosphorylation to Bak binding and drug resistance. Cancer Res. 2013, 73, 6998-7008. [CrossRef]

6. Delbridge, A.R.; Grabow, S.; Strasser, A.; Vaux, D.L. Thirty years of BCL-2: Translating cell death discoveries into novel cancer therapies. Nat. Rev. Cancer 2016, 16, 99-109. [CrossRef]

7. Li, Q.; Cheng, L.; Shen, K.; Jin, H.; Li, H.; Cheng, Y.; Ma, X. Efficacy and Safety of Bcl-2 Inhibitor Venetoclax in Hematological Malignancy: A Systematic Review and Meta-Analysis of Clinical Trials. Front. Pharmacol. 2019, 10, 697. [CrossRef]

8. Mihalyova, J.; Jelinek, T.; Growkova, K.; Hrdinka, M.; Simicek, M.; Hajek, R. Venetoclax: A new wave in hematooncology. Exp. Hematol. 2018, 61, 10-25. [CrossRef]

9. Deeks, E.D. Venetoclax: First Global Approval. Drugs 2016, 76, 979-987. [CrossRef]

10. Jonas, B.A.; Pollyea, D.A. How we use venetoclax with hypomethylating agents for the treatment of newly diagnosed patients with acute myeloid leukemia. Leukemia 2019, 33, 2795-2804. [CrossRef]

11. Juarez-Salcedo, L.M.; Desai, V.; Dalia, S. Venetoclax: Evidence to date and clinical potential. Drugs Context 2019, 8, 212574. [CrossRef]

12. Konopleva, M.; Pollyea, D.A.; Potluri, J.; Chyla, B.; Hogdal, L.; Busman, T.; McKeegan, E.; Salem, A.H.; Zhu, M.; Ricker, J.L.; et al. Efficacy and Biological Correlates of Response in a Phase II Study of Venetoclax Monotherapy in Patients with Acute Myelogenous Leukemia. Cancer Discov. 2016, 6, 1106-1117. [CrossRef]

13. Eyre, T.A.; Roeker, L.E.; Fox, C.P.; Gohill, S.H.; Walewska, R.; Walter, H.S.; Forconi, F.; Broom, A.; Arumainathan, A.; Brander, D.M.; et al. The efficacy and safety of venetoclax therapy in elderly patients with relapsed, refractory chronic lymphocytic leukaemia. Br. J. Haematol. 2020, 188, 918-923. [CrossRef] 
14. Al-Sawaf, O.; Zhang, C.; Tandon, M.; Sinha, A.; Fink, A.M.; Robrecht, S.; Samoylova, O.; Liberati, A.M.; Pinilla-Ibarz, J.; Opat, S.; et al. Venetoclax plus obinutuzumab versus chlorambucil plus obinutuzumab for previously untreated chronic lymphocytic leukaemia (CLL14): Follow-up results from a multicentre, open-label, randomised, phase 3 trial. Lancet Oncol. 2020, 21, 1188-1200. [CrossRef]

15. Freise, K.J.; Shebley, M.; Salem, A.H. Quantitative Prediction of the Effect of CYP3A Inhibitors and Inducers on Venetoclax Pharmacokinetics Using a Physiologically Based Pharmacokinetic Model. J. Clin. Pharmacol. 2017, 57, 796-804. [CrossRef]

16. Kaufman, J.L.; Gasparetto, C.; Schjesvold, F.H.; Moreau, P.; Touzeau, C.; Facon, T.; Boise, L.H.; Jiang, Y.; Yang, X.; Dunbar, F.; et al. Targeting BCL-2 with venetoclax and dexamethasone in patients with relapsed/refractory $\mathrm{t}(11 ; 14)$ multiple myeloma. Am. J. Hematol. 2021, 96, 418-427. [CrossRef]

17. Megias-Vericat, J.E.; Solana-Altabella, A.; Ballesta-Lopez, O.; Martinez-Cuadron, D.; Montesinos, P. Drug-drug interactions of newly approved small molecule inhibitors for acute myeloid leukemia. Ann. Hematol. 2020, 99, 1989-2007. [CrossRef]

18. Cheung, T.T.; Salem, A.H.; Menon, R.M.; Munasinghe, W.P.; Bueno, O.F.; Agarwal, S.K. Pharmacokinetics of the BCL-2 Inhibitor Venetoclax in Healthy Chinese Subjects. Clin. Pharmacol. Drug Dev. 2018, 7, 435-440. [CrossRef]

19. Kang, J.S.; Lee, M.H. Overview of therapeutic drug monitoring. Korean J. Intern. Med. 2009, 24, 1-10. [CrossRef]

20. Knezevic, C.E.; Clarke, W. Cancer Chemotherapy: The Case for Therapeutic Drug Monitoring. Ther. Drug. Monit. 2020, 42, 6-19. [CrossRef]

21. Gao, B.; Yeap, S.; Clements, A.; Balakrishnar, B.; Wong, M.; Gurney, H. Evidence for therapeutic drug monitoring of targeted anticancer therapies. J. Clin. Oncol. 2012, 30, 4017-4025. [CrossRef]

22. Bardin, C.; Veal, G.; Paci, A.; Chatelut, E.; Astier, A.; Leveque, D.; Widmer, N.; Beijnen, J. Therapeutic drug monitoring in cancer-are we missing a trick? Eur. J. Cancer 2014, 50, 2005-2009. [CrossRef]

23. de Jonge, M.E.; Huitema, A.D.; Schellens, J.H.; Rodenhuis, S.; Beijnen, J.H. Individualised cancer chemotherapy: Strategies and performance of prospective studies on therapeutic drug monitoring with dose adaptation: A review. Clin. Pharmacokinet. 2005, 44, 147-173. [CrossRef]

24. Eisenmann, E.D.; Jin, Y.; Weber, R.H.; Sparreboom, A.; Baker, S.D. Development and validation of a sensitive UHPLC-MS/MS analytical method for venetoclax in mouse plasma, and its application to pharmacokinetic studies. J. Chromatogr. B Analyt. Technol. Biomed. Life Sci. 2020, 1152, 122176. [CrossRef]

25. Liu, H.; Michmerhuizen, M.J.; Lao, Y.; Wan, K.; Salem, A.H.; Sawicki, J.; Serby, M.; Vaidyanathan, S.; Wong, S.L.; Agarwal, S.; et al Metabolism and Disposition of a Novel B-Cell Lymphoma-2 Inhibitor Venetoclax in Humans and Characterization of Its Unusual Metabolites. Drug Metab. Dispos. 2017, 45, 294-305. [CrossRef]

26. Choo, E.F.; Boggs, J.; Zhu, C.; Lubach, J.W.; Catron, N.D.; Jenkins, G.; Souers, A.J.; Voorman, R. The role of lymphatic transport on the systemic bioavailability of the Bcl-2 protein family inhibitors navitoclax (ABT-263) and ABT-199. Drug Metab. Dispos. 2014, 42, 207-212. [CrossRef]

27. Reddy, A.; Jadav, T.; Sahu, A.K.; Sengupta, P. LC-MS/MS bioanalytical method for quantification of binimetinib and venetoclax, and their pharmacokinetic interaction. Bioanalysis 2022, 14, 75-86. [CrossRef]

28. Roberts, A.W.; Davids, M.S.; Pagel, J.M.; Kahl, B.S.; Puvvada, S.D.; Gerecitano, J.F.; Kipps, T.J.; Anderson, M.A.; Brown, J.R.; Gressick, L.; et al. Targeting BCL2 with Venetoclax in Relapsed Chronic Lymphocytic Leukemia. N. Engl. J. Med. 2016, 374, 311-322. [CrossRef]

29. Minocha, M.; Zeng, J.; Medema, J.K.; Othman, A.A. Pharmacokinetics of the B-Cell Lymphoma 2 (Bcl-2) Inhibitor Venetoclax in Female Subjects with Systemic Lupus Erythematosus. Clin. Pharmacokinet. 2018, 57, 1185-1198. [CrossRef]

30. Schieber, M.; Ma, S. The expanding role of venetoclax in chronic lymphocytic leukemia and small lymphocytic lymphoma. Blood Lymphat. Cancer 2019, 9, 9-17. [CrossRef]

31. Izutsu, K.; Yamamoto, K.; Kato, K.; Ishikawa, T.; Fukuhara, N.; Terui, Y.; Choi, I.; Humphrey, K.; Kim, S.Y.; Okubo, S.; et al. Phase $1 / 2$ study of venetoclax, a BCL-2 inhibitor, in Japanese patients with relapsed or refractory chronic lymphocytic leukemia and small lymphocytic lymphoma. Int. J. Hematol. 2021, 113, 370-380. [CrossRef] [PubMed]

32. Deng, R.; Gibiansky, L.; Lu, T.; Agarwal, P.; Ding, H.; Li, X.; Kshirsagar, S.; Lu, D.; Li, C.; Girish, S.; et al. Bayesian Population Model of the Pharmacokinetics of Venetoclax in Combination with Rituximab in Patients with Relapsed/Refractory Chronic Lymphocytic Leukemia: Results from the Phase III MURANO Study. Clin. Pharmacokinet. 2019, 58, 1621-1634. [CrossRef] [PubMed]

33. Jones, A.K.; Freise, K.J.; Agarwal, S.K.; Humerickhouse, R.A.; Wong, S.L.; Salem, A.H. Clinical Predictors of Venetoclax Pharmacokinetics in Chronic Lymphocytic Leukemia and Non-Hodgkin's Lymphoma Patients: A Pooled Population Pharmacokinetic Analysis. AAPS J. 2016, 18, 1192-1202. [CrossRef] [PubMed]

34. Ramanathan, R.K.; Egorin, M.J.; Takimoto, C.H.; Remick, S.C.; Doroshow, J.H.; LoRusso, P.A.; Mulkerin, D.L.; Grem, J.L.; Hamilton, A.; Murgo, A.J.; et al. Phase I and pharmacokinetic study of imatinib mesylate in patients with advanced malignancies and varying degrees of liver dysfunction: A study by the National Cancer Institute Organ Dysfunction Working Group. J. Clin. Oncol. 2008, 26, 563-569. [CrossRef]

35. Salem, A.H.; Dave, N.; Marbury, T.; Hu, B.; Miles, D.; Agarwal, S.K.; Bueno, O.F.; Menon, R.M. Pharmacokinetics of the BCL-2 Inhibitor Venetoclax in Subjects with Hepatic Impairment. Clin. Pharmacokinet. 2019, 58, 1091-1100. [CrossRef]

36. FDA; CDER; CVM. Guidance for Industry. Bioanalytical Method Validation. 2018. Available online: http://www.fda.gov/ downloads/drugs/guidances/ucm070107.pdf (accessed on 17 December 2021). 